\title{
Activités
}

3-1 | avril 2006

Varia

\section{Psychologie de l'art, de Lev S. Vygotski}

Traduit du russe par Françoise Sève. Paris : La Dispute, 2005, 382 pages

\section{Gabriel Fernandez}

\section{(2) OpenEdition}

Journals

Édition électronique

URL : http://journals.openedition.org/activites/1883

DOI : 10.4000/activites.1883

ISSN : 1765-2723

Éditeur

ARPACT - Association Recherches et Pratiques sur les ACTivités

Référence électronique

Gabriel Fernandez, «Psychologie de l'art, de Lev S. Vygotski », Activités [En ligne], 3-1 | avril 2006, mis en ligne le 02 avril 2006, consulté le 22 septembre 2020. URL : http://journals.openedition.org/activites/ 1883 ; DOI : https://doi.org/10.4000/activites.1883

\section{(c) (i) (9)}

Activités est mis à disposition selon les termes de la licence Creative Commons Attribution - Pas d'Utilisation Commerciale - Pas de Modification 4.0 International. 


\section{Note de lecture par Gabriel Fernandez}

Psychologie de l'art, de Lev S. Vygotski, traduit du russe par Françoise Sève. Paris: La Dispute, $2005,382 \mathrm{p}$.

Rédigée en 1925, publiée en russe en 1965, puis traduit en plusieurs langues jusqu'en 1976, la Psychologie de l'art de Lev Vygotski est enfin disponible en français. Ce travail du fondateur de la conception «historico-culturelle» du psychisme humain situe, dès cette époque, la psychologie en amont de l'idéologie, reconnaissant ainsi une certaine autonomie de la première relativement à la seconde. Il s'agit pour Vygotski d'expliquer pourquoi l'œuvre d'art continue d'émouvoir les humains en différents lieux et aux différentes époques, alors que les conditions historiques et matérielles de son élaboration n'ont plus cours. Autrement dit, il s'agit de trouver la loi psychologique sur laquelle l'œuvre d'art est fondée et le mécanisme par lequel elle agit sur l'activité psychique. Ce qui revient à expliquer la fonction psychologique de l'art. Selon la conception de Vygotski, la psychologie de l'art ne peut être qu'une psychologie pure et impersonnelle, indépendante de l'auteur comme du lecteur/spectateur, c'est-à-dire, une psychologie qui ne considère que la forme et le matériau de l'œuvre.

La seule méthode adéquate à cet objet doit être objective et analytique : elle part de l'œuvre d'art pour arriver à une synthèse psychologique. En effet, l'art est cette activité proprement humaine qui systématise la sphère des sentiments, sphère du psychisme de l'homme social. Dès lors, la réaction esthétique doit être reconstituée dans son intégralité impersonnelle à partir des données objectives. La psychologie de l'art s'intéresse à la réaction impersonnelle, qui n'est propre à aucun individu concret.

Ni le scientisme du behaviorisme ou de la réflexologie, ni l'idéalisme subjectiviste, ne répondent complètement à la question. Par-delà leurs différences importantes et leurs contributions à la connaissance des processus psychologiques, la critique de Vygotski montre en quoi ces deux courants théoriques ont en commun d'être unilatéraux. Le premier reconnaît à l'art la propriété d'être un moyen de connaissance en tant que travail de la pensée. Mais il laisse de côté le rôle des émotions dans ce travail en les considérant comme des éléments fortuits au sein de ce processus. A l'inverse, le sensualisme accorde aux émotions le rôle déterminant, cherchant à proposer une typologie des émotions permettant de distinguer les émotions artistiques des autres catégories d'émotions. La pensée n'est plus ici que le contrecoup du vécu émotionnel.

La psychanalyse, par contre, a fait selon Vygotski cette importante découverte que l'art est transformation de l'inconscient en des formes sociales, c'est-à-dire des comportements ayant un sens social et des fins assignées. Mais, en 1925, date à laquelle Vygotski écrit son essai, elle n'a pas fait de cette découverte le moyen d'élucider la fonction psychologique de l'art. A cause, note-t-il, de la sous-estimation de la sphère consciente et de son rôle actif et autonome. Jusqu'alors, pour la psychanalyse, la jouissance est davantage fondée sur le contenu que sur la forme de l'œuvre, et du coup l'analyse de la forme et de sa fonction psychologique est délaissée, autant qu'est négligé l'examen des changements historiques de cette fonction. Pourtant, l'art en tant que résolution sociale de l'inconscient est, selon Vygotski, la réponse la plus probable à la question de l'art du point de vue de l'inconscient.

Vygotski centre alors son analyse sur la forme de l'œuvre après avoir constaté, avec les formalistes, que quand cette forme est détruite, la réaction esthétique est perdue. Le matériau de l'art est tout ce que l'artiste trouve tout prêt (mots, sons, images...), tandis que la forme, résultat de l'activité de l'artiste, est le mode d'exposition du matériau en vue de créer une réaction esthétique. La forme est donc le procédé artistique et son résultat. Malgré ce primat accordé à la forme, qui a tant intéressé le 
cinéaste Eisenstein, il n'en critique pas moins les formalistes russes qui pensent pouvoir opposer un principe anti-psychologique à l'étude de toute œuvre d'art. Vygotski rejette l'hypothèse selon laquelle la forme serait le but ultime de l'activité artistique car pour lui la forme n'est pas à ellemême sa propre fin. Il cherche donc à résoudre la question du sens et de la structure de la forme artistique en tenant compte des changements historiques des contenus mêmes des œuvres d'art.

Et de se mettre à l'analyse de la réaction esthétique à propos de trois genres littéraires, la fable (Krylov), la nouvelle (Bounine) et la tragédie (Shakespeare). «L'idée centrale de la psychologie de l'art, affirme Vygotski, consiste à reconnaître que la forme artistique l'emporte sur le matériau, ou, ce qui revient au même, que l'art est une technique sociale du sentiment ». Pour Vygotski, c'est la « contradiction affective » qu'organise la forme qui importe. Ainsi, la tragédie de Shakespeare metelle en scène la ligne droite du récit, laquelle est constamment percutée par la ligne sinueuse des obstacles que dresse la composition de la pièce. Le sens de ces sinuosités est révélé par la scène finale traitée sur deux plans psychologiques opposés. D'où cette loi de la réaction esthétique : un affect qui se développe dans deux directions opposées et qui, à son point culminant, est réduit à néant en une espèce de court-circuit.

C'est ce que l'auteur nomme, renouvelant la définition d'Aristote, la « catharsis ». Pour Vygotski, toute œuvre d'art recèle une contradiction affective qui mène au court-circuit entre des affects opposés et à l'anéantissement de la réaction externe. La base de ce processus est l'abolition du contenu par la forme. La formule psychologique de l'art peut donc s'énoncer de la façon suivante : l'œuvre d'art provoque des affects vécus dans leur réalité mais déchargeant leur énergie dans l'activité d'imagination. L'aspect moteur est inhibé, donnant l'impression d'avoir affaire à des sentiments illusoires. C'est sur cette unité du sentiment et de l'imagination que repose tout art, car l'imagination et le sentiment sont un seul et même processus, au sein duquel l'imagination n'est que l'expression centrale de la réaction émotionnelle. L'activité psychique ainsi affectée transforme les sentiments en se servant des émotions à titre de moyens de l'activité.

L'essai se termine sur l'examen des rapports entre l'art et la vie. L'art implique quelque chose en soi qui transcende le sentiment ordinaire. Il tire de la vie son matériau, mais il ajoute une plus-value qui n'est pas contenue dans le matériau : la transformation du sentiment individuel en une forme sociale grâce au procédé artistique qui organise une lutte entre la forme et le contenu. L'art est donc une technique sociale du sentiment, c'est-à-dire, un sentiment social partagé. Il met à contribution une part du psychisme qui ne trouve pas à s'extérioriser dans la vie quotidienne. Partant des sentiments que provoque la vie habituelle, l'art les soumet à une réélaboration au moment de la catharsis. Le rapport au réel n'est donc pas à chercher du côté d'un reflet du social mais dans le processus même d'une catharsis. À travers le lien cathartique de l'inconscient et du social en art, le réel est produit par le sujet plutôt que reflété.

Cependant, l'art n'engendre pas l'action, il ne fait que préparer l'organisme à l'action. Freud fait remarquer que ce qui est utile quand un homme a peur et fuit, c'est l'action de fuir. En art, c'est l'inverse : c'est la peur qui est utile parce qu'elle crée la possibilité d'une fuite ou d'une attaque. Pour Vygotski, l'art complète alors le cercle des vies réalisées en élargissant ses possibilités. En influençant les sentiments, l'art influence la volonté puisqu'un sentiment est une volonté en germe. Il le fait de façon complexe du fait qu'entre l'homme et le monde il y a le milieu social qui réfracte et oriente toute excitation agissant du dehors, ainsi que toute action dirigée vers l'extérieur. Tout ce que l'art provoque comme activité d'un sujet, il le fait dans son corps et par son intermédiaire, notamment par l'intermédiaire des variations du tonus musculaire et des attitudes, ou encore par la variation des rythmes viscéraux, en particulier cardiaque et surtout respiratoire. C'est pourquoi l'acte artistique ne peut pas se ramener à des opérations purement conscientes. Mais la direction donnée aux processus conscients influence les processus inconscients.

Finalement, on peut dire de l'essai de Vygotski qu'il permet de mieux comprendre le développement subjectif, inconscient compris, en ce qu'il découvre la loi psychologique de la mise en mouvement 
des sentiments du spectateur provoquée par la forme artistique. Qui s'intéresse à l'activité humaine, quand bien même il ne s'agirait que de l'activité professionnelle, sait d'expérience combien ces mouvements subjectifs sont au cœur de ce qu'il cherche à expliquer, voire à transformer. La lecture de Psychologie de l'art permettra sans nul doute de mieux les appréhender, et ainsi de les mettre au service de l'intervention. 